\title{
Lighter-Than-Air Wind Energy Systems: Stability Analysis
}

\author{
Dhruv Suri, ${ }^{1, \text { a) }}$ Amartya Mukherjee, ${ }^{1}$ Raahil Nayak, ${ }^{1}$ Jayakrishnan \\ Radhakrishnan, ${ }^{1}$ and NG Yin Kwee ${ }^{2}$ \\ ${ }^{1)}$ Department of Aeronautical and Automobile Engineering, Manipal Institute of Technology, Manipal Academy of Higher \\ Eduction, Manipal, Karnataka 576104, India. \\ ${ }^{2}$ School of Mechanical and Aerospace Engineering, Nanyang Technological University, 50 Nanyang Avenue, Singapore 639798 \\ a) Corresponding author: dhruv.suri@learner.manipal.edu
}

\begin{abstract}
Stability analysis of a lighter-than-air wind energy system is carried out by solving the Reynolds Averaged NavierStokes (RANS) equations in two dimensions using commercial CFD solver ANSYS Fluent ${ }^{\circledR}$. Not disregarding experimental and high fidelity methods, the present analysis is characterized by a low computational cost and high accuracy. The lighter-than-air system is represented by an aerodynamic shell while the rotor is modelled as an actuator disk (AD). The stability of the lighterthan-air system has been investigated on the basis of blowdown angle. The design space has been constructed by following a brute force selection approach. Physical changes in the flowfield due to varying shell camber and thickness have been discussed. Results demonstrate that an increase in both camber and thickness significantly affect the stability characteristics of the shell. This objective of the present study is to discuss the interplay between shell cross-section geometry and blowdown angle.
\end{abstract}

\section{INTRODUCTION}

The impetus towards sustainable energy production has led to the development of the sector, with the present global generation capacity at $486 \mathrm{GW}$ (2018), increasing by $12.5 \%$ as compared to the previous year [1]. In order for wind technology to be a viable contender, next generation wind turbines should increase both performance and reliability, thus lowering the levelized cost of electricity (LCOE). Conventional ground based wind turbines do not have access to consistent and strong winds and incur substantial costs due to reinforcement structures and added material consumption. A surge in innovation and development has paved the way for a relatively new area of focus: airborne wind energy systems (AWES).

AWES are able to harvest energy from strong and consistent winds at high altitudes while offsetting substantial costs owing to reduced material consumption [2]. This low-cost method of renewable energy production can be utilized in regions affected by natural disasters, remote areas not connected to an electrical grid as well as to counterbalance the high energy demand of densely populated regions. AWES are connected to the ground by means of a tether which may conduct electricity depending on the configuration of the system employed. Ground-Gen or GG-AWES use the aerodynamic forces generated in-flight to convert mechanical energy in the form of tension applied on the tether into electrical energy using a ground based generator. Gliders and kites constitute the most common GG-AWES and have been studied by a number of researchers across the world [3]. Contrariwise, Fly-Gen or FG-AWES are characterized by on-board energy production.

The most common lighter-than-air aerial wind turbine studied in literature is the Altaeros BAT developed by Altaeros Energies [4] [5]. Buoyant shells such as the BAT rely on aerostatic lift to operate at a desired altitude above the ground; however, the effect of drag on the shell is pronounced due to the prevalence of high mean wind speeds at operational altitudes. These drag forces make the airborne wind turbine susceptible to blowdown which can be prevented by increasing the resultant lift provided by the shell [6]. To this aim, the design of an airborne wind turbine must take into account both the aerodynamic as well as stability constraints imposed by the operating conditions.

Shell based airborne wind turbines are analogous to ground based ducted wind turbines (DWTs) in terms of operating principle. In contrast to lighter-than-air based energy systems, there is a significant amount of literature on DWTs based on analytical, numerical and experimental methods to characterize the aerodynamic performance [7] [8] [9] [10]. Airfoil based duct cross-sections augment the flow through the throat of the duct thereby increasing the mass flow rate incident on the rotor plane. Bontempo and Manna [11] studied the effect of duct cross section geometry on the performance of ducted propulsion systems for aeronautical applications using a semi-analytical model. They found that the cross-sectional camber up to a certain limit leads to an increase in the performance of the enclosed rotor, however the semi-analytical model does not take into account the mutual interaction between the shell (or duct) and the rotor. The suitability of a 2D RANS approach for analyzing the performance of a DWT was corroborated by Dighe et al [12] and Suri et al [13]. They found that a numerical model based on the RANS method can accurately predict the aerodynamic performance of ducted systems. 
The current work uses an incompressible RANS method using commercial CFD solver ANSYS Fluent ${ }^{\circledR}$. Not disregarding the experimental and high fidelity methods, the present analysis is characterized by a low computational cost and high accuracy. The scope of the present analysis is to determine the stability of the shell configuration in terms of the blowdown angle $(\boldsymbol{\delta})$ which is explained in subsection. This paper thus discusses the interplay between shell cross-section geometry and blowdown while also presenting an overview of the changes in global performance coefficients.

\section{COMPUTATIONAL METHODOLOGY}

\section{Actuator Disk Flow Model}

The incompressible flow across a horizontal axis wind turbine (HAWT) is modelled as an AD with constant loading. The pressure drop across the AD is determined using a semi - empirical relation between the thrust coefficient curve and the velocity at the disk. The $\mathrm{AD}$ exerts a constant thrust force per unit surface $T_{A D}$, which corresponds to a non-dimensional thrust force coefficient $C_{T, A D}$ :

$$
C_{T, A D}=\frac{T_{A D}}{\frac{1}{2} \rho U_{\infty}^{2} S_{A D}}
$$

where $\rho$ is the fluid density $U_{\infty}$ is the free stream velocity, $S_{A D}$ is the surface area of the AD.

For an FG-AWE system, the aerodynamic shell exerts an added thrust force $F_{\text {shell }}$ in addition to the thrust force $T_{A D}$ exerted by the AD. The total pressure drop across the AD will take into account the thrust force exerted by the shell, which corresponds to a non-dimensional shell thrust force coefficient $C_{T, \text { shell }}$ :

$$
C_{T, \text { shell }}=\frac{F_{\text {shell }}}{\frac{1}{2} \rho U_{\infty}{ }^{2} S_{A D}}
$$

The presence of $F_{\text {shell }}$ results in an additional mass flow rate $\triangle \dot{m}=\rho S_{A D} U_{A D}$ across the AD plane. It is important to note that although the thrust force coefficient $C_{T, A D}$ is assumed to be uniform across the $\mathrm{AD}$, the axial velocity at the $\mathrm{AD}$ cannot be regarded as uniform. The mean velocity $U_{A D}$ at the $\mathrm{AD}$ is the spatial average of the local stream wise velocity at the AD plane:

$$
U_{A D}=\frac{1}{S_{A D}} \int_{r a d} U_{x} d S
$$

where $U_{x}$ is the differential axial velocity term across the AD surface $d S$ in the freestream direction $x$.

The total thrust exerted by the buoyant shell is the algebraic sum of the thrust exerted by the shell and the AD thrust:

$$
T=T_{A D}+T_{\text {shell }}
$$

The above equation can be formulated in terms of a non-dimensional thrust force coefficient:

$$
C_{T}=C_{T, A D}+C_{T, \text { shell }}
$$

The total power coefficient of a buoyant shell will take into account the power coefficient of both the shell and the $\mathrm{AD}$. This leads to a power coefficient of the flow model using AD surface area $S_{A D}$ as the reference area:

$$
C_{P}=\frac{P}{\frac{1}{2} \rho U_{\infty}{ }^{2} S_{A D}}=\frac{U_{A D}}{U_{\infty}} C_{T}
$$

$C_{T, \text { shell }}$ and mean velocity $U_{A D}$ are not known a priori and are obtained on post processing the results of the numerical simulation. 


\section{Computational Settings and Parameters}

A commercial CFD solver ANSYS Fluent ${ }^{\circledR}$ has been used for a complete viscous unsteady solution of incompressible flow around the buoyant shell. The governing flow equations are the Reynolds-averaged Navier Stokes (RANS) equations. The distance from the $\mathrm{AD}$ location to the domain inlet and outlet are $12 c$ and $24 c$, respectively. The computational grid consists of quadrilateral cells with minimum $y^{+}$value of 1 on the shell walls. Boundary conditions are: a uniform velocity at the inlet, zero gauge static pressure at the outlet, no-slip walls for shell surfaces. A symmetric boundary condition is applied along the center-line axis while a fan boundary condition is used for the AD. The k- $\omega$ SST (shear stress transport) model is used as turbulence model.

\section{Verification and Validation}

RANS solutions are sensitive to the discretization of the computational domain. For the present computations, a Cgrid structured zonal approach is chosen, which proved advantageous in the case of a curved boundary, i.e. the shell's leading edge. The $\mathrm{C}$-shaped loop terminates in the wake region. In order to obtain a solution independent of the grid size, three grids with varying refinement (fine, medium and coarse) are chosen. The refinement factor in each direction is 2. The variation of mean velocity at the actuator disk $U_{A D}$ is computed for the NACA 5415 profile for $U_{\infty}=10 \mathrm{~m} / \mathrm{s}$ and $C_{T, A D}=0.7$. The results for the grid independence analysis are presented in Table I. The medium grid consisting of 340230 elements is selected for the numerical analysis.

TABLE I. Grid statistics for grid independence study of NACA 5415 profile.

\begin{tabular}{|c|c|c|}
\hline Grid & Number of cells & $\frac{U_{A D}}{U \infty}$ \\
\hline Coarse & 150105 & 0.98140 \\
\hline Medium & 340230 & 0.96151 \\
\hline Fine & 761028 & 0.96152 \\
\hline
\end{tabular}

Due to the lack of experimental data on FG-AWES, the computational study conducted by Saleem and Kim [14] is used as a reference study for validation. A non-uniform loading has been imposed on the AD as opposed to that presented in Equation 1. The pressure drop across the AD is again defined using Equation 1.

In Figure 1, the mass flow rate $\dot{m}$ is plotted as a function of free-stream velocity $U_{\infty}$. A good agreement between the two CFD-simulations is found. Minor variations between the two studies may be due to 3D effects such as rotor wake interaction which are not accounted for in a 2D analysis. Nevertheless, the trend for $\dot{m}$ and $U_{\infty}$ obtained using a 2D computational model is consistent with that obtained using high fidelity 3D simulations, thereby validating the applicability of the setup to analyze the aerodynamic performance of different shell configurations.

\section{BLOWDOWN EFFECT}

A number of constraints must be considered while designing a lighter-than-air wind energy system. Buoyant shells rely on aerostatic lift to remain suspended in the air. This aerostatic lift or force due to buoyancy can be measured as a function of the volume of the shell and the relative densities of the surrounding air and the lighter-than-air gas:

$$
F_{b}=V\left(\rho_{a i r}-\rho_{g}\right)
$$

where $F_{b}$ is the force due to buoyancy, $V$ is the volume of the shell, $\rho_{a i r}$ is the relative density of the atmospheric air at operating conditions and $\rho_{g}$ is the relative density of the lighter than air gas enclosed within the shell.

Buoyant shells are also susceptible to a drag force $F_{D}$ which acts in a direction normal to $F_{b}$. The effect of this drag force is pronounced at high wind speeds which are prevalent at the operational altitudes of airborne wind turbines.

To overcome the effect of blowdown due to $F_{D}$, AWES require design investigations from both an aerodynamic as well as a stability perspective. Shell configurations are thus composed of airfoil cross-sections thereby generating an 


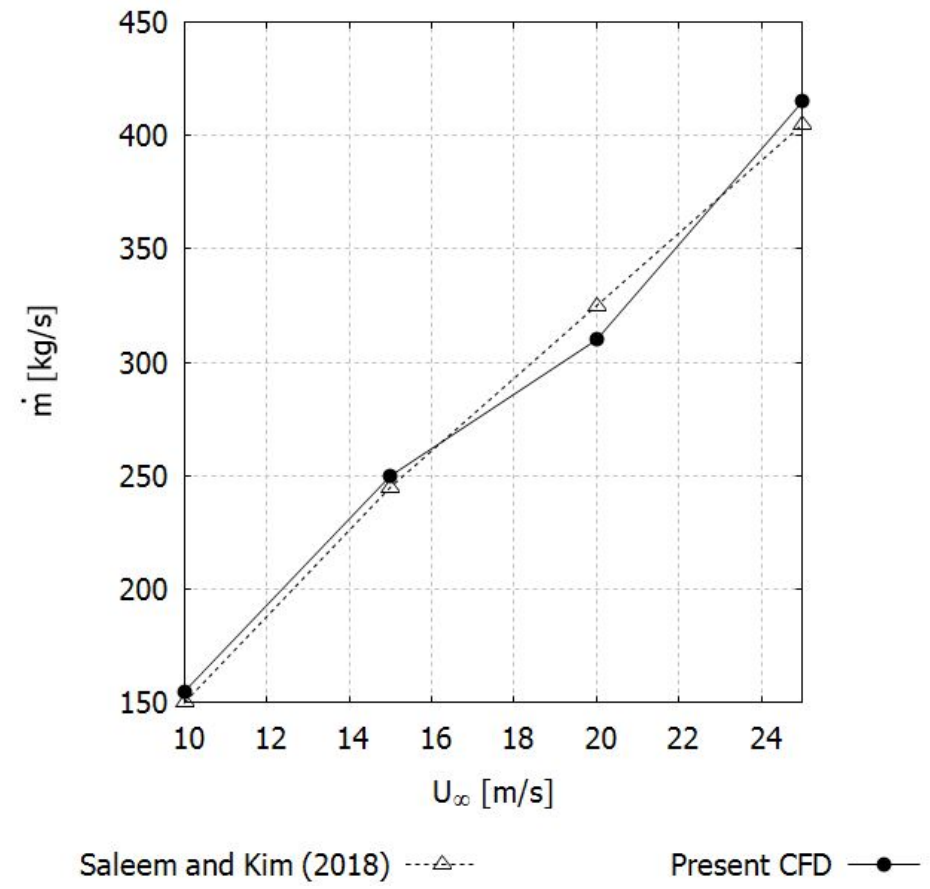

FIGURE 1. Variation in mass flow rate $m$ is as a function of free-stream velocity $U_{\infty}$

aerodynamic lift $F_{l}$. The total lift $F_{L}$ produced by a buoyant shell is the resultant of aerostatic lift $F_{b}$, aerodynamic lift $F_{l}$ and weight of the system $m g$ :

$$
F_{L}=F_{b}+F_{l}-m g
$$

where $m$ is the mass of the AWE system and $g$ is the acceleration due to gravity.

The blowdown angle $\delta$ is determined by the lift/drag ratio of the lighter-than-air system:

$$
\delta=\arctan \frac{F_{D}}{F_{L}}=\arctan \left(\frac{\frac{1}{2} \rho_{h} V_{\infty}^{2} C_{D} S_{A D}}{F_{e b}+\frac{1}{2} \rho_{h} V_{\infty}^{2} C_{D} S_{A D}}\right)
$$

where $F_{e b}$ represents the excess buoyant force due to the enclosed lighter-than-air gas and is dependent on the weight of the AWE system. As the scope of the present paper is limited to discussing the aerodynamic performance and stability of buoyant shell configurations, we neglect the effect of $F_{e b}$ and assume that $F_{e b}$ solely balances the weight of the AWE system at the operational altitude.

Equation 9 can then be re-written as:

$$
\delta=\arctan \frac{C_{D}}{C_{L}}
$$

A blowdown angle $\delta=45^{\circ}$ represents a critical angle wherein $C_{L}=C_{D}$. When $C_{D}>C_{L}, \delta>45^{\circ}$ and the system is unstable. Contrariwise, when $C_{D}<C_{L}, \delta<45^{\circ}$ and the system can be said to be stable. In the present study, effect of varying the shell cross-section geometry and its impact on $\delta$ is investigated. As the analysis is $2 \mathrm{D}$, the simulations may under-predict $F_{D}$ and consequently $\delta$. Nevertheless, a good approximation of the effect of $\frac{C_{L}}{C_{D}}$ on $\delta$ can be made.

\section{RESULTS AND DISCUSSION}

Figure 2 illustrates the correlation between the blowdown angle $\delta$ and freestream velocity $U_{\infty}$ obtained using Equation 10. In general, $\delta$ increases linearly for low $U_{\infty}$. Subsequently, $\delta$ stabilizes at a certain maxima which varies with shell cross-section camber and thickness. As $\delta$ is a univariate function of $\frac{C_{L}}{C_{D}}$, two noticeable inferences can be drawn. 
Firstly, the magnitude of $\delta$ is reduced for the shell configurations where a high-lift airfoil is employed. With increasing camber, $\frac{C_{L}}{C_{D}}$ increases and hence $\delta$ is smaller as compared to geometries without camber. A similar trend is observed for shell configurations with varying thickness. While considering standalone airfoil geometries not coupled with a rotor, the NACA 9405 profile is characterized by a higher L/D ratio as compared to the NACA 9415 and NACA 9425 profiles. This trend is observed while comparing the L/D ratios and the $\delta$ of the shell configurations. In fact, the value of $\delta$ obtained for the NACA 9405 shell configuration is lower than that for all profiles considered.

Secondly, Figure 2 shows that the AWE system is stable for all shell configurations considered, as $\delta<45^{\circ}$. The maximum blowdown angle achieved is $41^{\circ}$ by the NACA 1415 configuration while the minimum angle is $20^{\circ}$ by the NACA 9405 configuration.

The results obtained from the present analysis also pave the way for some general comments on the stability characteristics of lighter-than-air FG-AWE systems. While the magnitude of $\delta$ for the analyzed shell profiles may seem inconsequential at first, it is important to note that the operational altitude of the system and hence the length of the tether is set to $400 \mathrm{~m}$. With blowdown, there is a displacement of the AWES in both the horizontal and vertical direction, and hence a decrease in the operational altitude of the system. The vertical displacement $v_{x}$ downstream of the ground station is given by:

$$
v_{x}=L_{t} \cos (\delta)
$$

where $L_{t}$ is the original operational altitude of the AWES $(\mathrm{h}=400 \mathrm{~m})$.

For the present study, the critical blowdown angle at $\delta=45^{\circ}$ corresponds to $v_{x}=210 \mathrm{~m}$, which is seen to be a rather large deflection. If we consider the NACA 9405 shell profile with $\delta=20^{\circ}$, the resultant $v_{x}=163 \mathrm{~m}$. Thus, deflection of the AWES downstream of the ground station can be evaluated in terms of $\delta$ and is an important parameter that affects the global performance of the shell. While the five NACA shell profiles were selected in a brute-force manner, they do give us insight into the role of L/D ratio on the blowdown angle and horizontal deflection. While designing a lighterthan-air AWES from a system stability perspective, slender profiles with high camber yield the best performance in terms of downstream displacement.

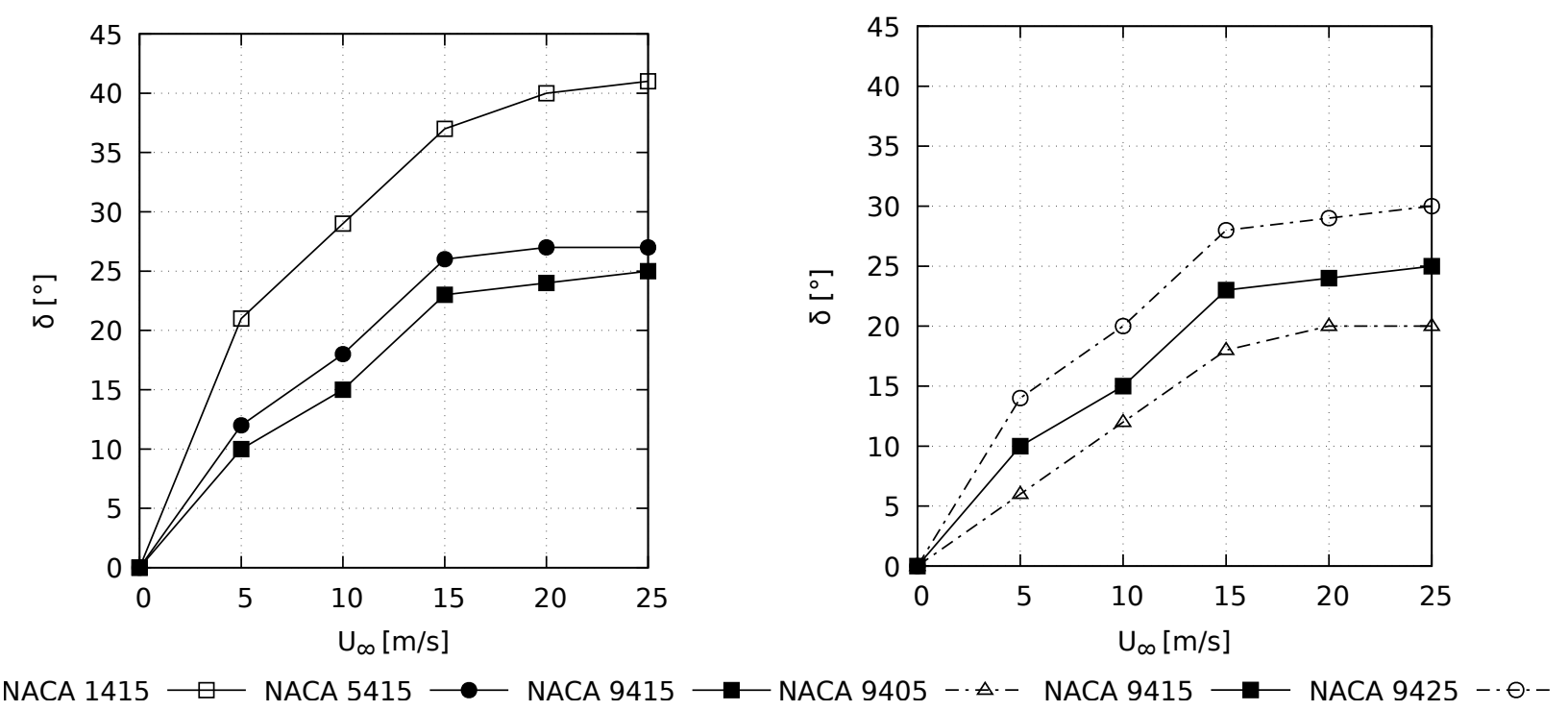

FIGURE 2. Blowdown angle $\delta$ vs freestream velocity $U_{\infty}$ to investigate the effect of (top) camber and (bottom) thickness, respectively

\section{CONCLUSION}

The performance and flowfield of five different shell geometries (NACA 1415, NACA 5415, NACA 9405, NACA 9415, NACA 9425) of a lighter-than-air wind energy system has been analyzed using a RANS method. The shell 
configuration is represented by a 2D airfoil and the rotor is modelled as an actuator disk (AD). The NACA 9405 profile is characterized by the lowest blowdown angle $\delta=20^{\circ}$. The cross-section geometry of the buoyant shell is responsible for inducing a greater mass flow rate $(m)$ across the AD thereby increasing the thrust force of the system, which further helps counter-balance the drag force.

The results obtained by the present study can be summarized as follows:

1. The presented RANS validation results indicate that the shell-AD model is very efficient in providing reliable results for analyzing the aerodynamic and stability characteristics of a buoyant wind turbine

2. $\delta$ is affected by the cross-sectional camber and thickness of the shell configuration: shell profiles with high camber and low thickness correspond to greater stability characteristics. $\delta$ is reduced for shell configurations where a high-lift airfoil is employed. The maximum blowdown angle achieved is $41^{\circ}$ by the NACA 1415 profile while the minimum angle is $20^{\circ}$ by the NACA 9405 profile.

3. The presence of an aerodynamic shell around the rotor augments the flow passing through the rotor plane, thereby increasing the performance of the HAWT.

4. While designing a lighter-than-air wind energy system, both aerodynamic and aerostatic lift should be taken into account. Moreover, the effect of induced drag on the buoyant system must not be neglected

The present study evaluates the stability of a lighter-than-air wind energy system in terms of blowdown and the effect of shell cross-sectional geometry on the performance of the system. It is likely worthwhile to conduct an optimization of both the stability and global performance coefficients using a multi-objective approach instead of a brute-force selection. The former approach could yield a favorable design from both an aerodynamic as well as stability perspective.

\section{REFERENCES}

1. E. Dupont, R. Koppelaar, and H. Jeanmart, "Global available wind energy with physical and energy return on investment constraints," Applied Energy 209, 322-338 (2018).

2. Z. Ye, H. Lawner, I. Chaer, and M. Ross, "Viability of airborne wind energy in the united kingdom," Journal of Thermal Science and Engineering Applications, 1-43 (2019).

3. A. Cherubini, A. Papini, R. Vertechy, and M. Fontana, "Airborne wind energy systems: A review of the technologies," Renewable and Sustainable Energy Reviews 51, 1461-1476 (2015).

4. C. Vermillion, T. Grunnagle, and I. Kolmanovsky, "Modeling and control design for a prototype lighter-than-air wind energy system," in 2012 American Control Conference (ACC) (IEEE, 2012) pp. 5813-5818.

5. C. Vermillion, B. Glass, and A. Rein, "Lighter-than-air wind energy systems," in Airborne Wind Energy (Springer, 2013) pp. 501-514.

6. R. Schmehl, Airborne wind energy: advances in technology development and research (Springer, 2018).

7. G. J. Van Bussel, "The science of making more torque from wind: Diffuser experiments and theory revisited." in Journal of Physics: Conference Series, Vol. 75 (IOP Publishing, 2007) p. 012010.

8. V. Dighe, D. Suri, F. Avallone, and G. van Bussel, "Ducted wind turbines in yawed flow: A numerical study," .

9. J. Tang and G. J. van Bussel, "Wind tunnel testing airfoil with screens at low reynolds number," in 2018 Wind Energy Symposium (2018) p. 0995.

10. D. Suri, "The potential for ducted wind turbines in an urban environment," (2019).

11. R. Bontempo and M. Manna, "Performance analysis of open and ducted wind turbines," Applied Energy 136, 405-416 (2014).

12. V. Dighe, F. Avallone, and G. van Bussel, "Computational study of diffuser augmented wind turbine using actuator disc force method," International Journal of Computational Methods and Experimental Measurements 4, 522-531 (2016).

13. D. Suri, "Design of an optimized inlet shroud for a flanged diffuser," (2019).

14. A. Saleem and M.-H. Kim, "Aerodynamic analysis of an airborne wind turbine with three different aerofoil-based buoyant shells using steady rans simulations," Energy Conversion and Management 177, 233-248 (2018).

15. V. V. Dighe, G. de Oliveira, F. Avallone, and G. van Bussel, "On the effects of the shape of the duct for ducted wind turbines," in 2018 Wind Energy Symposium (2018) p. 0997.

16. J. Radhakrishnan and D. Suri, "Design and optimisation of a low reynolds number airfoil for small horizontal axis wind turbines," in $I O P$ Conference Series: Materials Science and Engineering, Vol. 377 (IOP Publishing, 2018) p. 012053.

17. O. De Vries, "Fluid dynamic aspects of wind energy conversion," Tech. Rep. (Advisory Group for Aerospace Research and Development NEUILLY-SUR-SEINE (France), 1979). 